\title{
The Lagrangian, Self-Adjointness, and Conserved Quantities for a Generalized Regularized Long-Wave Equation
}

\author{
Long Wei and Yang Wang \\ Department of Mathematics, Hangzhou Dianzi University, Zhejiang 310018, China \\ Correspondence should be addressed to Long Wei; alongwei@gmail.com
}

Received 9 January 2014; Accepted 2 May 2014; Published 8 May 2014

Academic Editor: Tiecheng Xia

Copyright ( 2014 L. Wei and Y. Wang. This is an open access article distributed under the Creative Commons Attribution License, which permits unrestricted use, distribution, and reproduction in any medium, provided the original work is properly cited.

\begin{abstract}
We consider the Lagrangian and the self-adjointness of a generalized regularized long-wave equation and its transformed equation. We show that the third-order equation has a nonlocal Lagrangian with an auxiliary function and is strictly self-adjoint; its transformed equation is nonlinearly self-adjoint and the minimal order of the differential substitution is equal to one. Then by Ibragimov's theorem on conservation laws we obtain some conserved qualities of the generalized regularized long-wave equation.
\end{abstract}

\section{Introduction}

The generalized regularized long-wave (GRLW) equation

$$
u_{t}+u_{x}+a u^{m} u_{x}+b u_{x x t}=0
$$

was first put forward as a model for small-amplitude long waves on the surface of water in a channel by Peregrine $[1,2]$, where $m$ is a positive integer, and $a$ and $b$ are two constants. It is an alternative description of nonlinear dispersive waves to the Korteweg de Vries equation [1] and has been used to describe phenomena with weak nonlinearity and dispersion waves, including nonlinear transverse waves in shallow water, ion-acoustic and magnetohydrodynamic waves in plasma and phonon packets in nonlinear crystals, pressure waves in liquid-gas bubble mixture, rotating flow down a tube, and lossless propagation of shallow water waves; see [3] and the references therein.

Equation (1) has been studied extensively in the past both analytically and numerically by means of, for example, the Adomian decomposition method, the Fourier method, the finite difference method, the finite element method, the variational iteration method, the mesh-free method, the cubic B-spline collocation procedure, the sine-cosine method, and the double reduction method [4-11], and so forth. A few of the conserved quantities by using the 1soliton solution and solitary-wave solution of the $R(m, n)$ equation were calculated in [12]. In [11], the authors employed the Lie symmetry method and double reduction theory to obtain some exact solutions and derived the conservation laws for (1) via the so-called "partial Noether approach" after increasing its order. Noting that the GRLW equation is a third-order partial differential equation and it is difficult to obtain a variational formulation/Lagrangian for (1) by a classical approach, the authors converted the equation to the following fourth-order equation by assuming new dependent variable $v$ to be the derivative of original dependent variable $u$ by setting $u=v_{x}$ as follows:

$$
v_{x t}+v_{x x}+a v_{x}^{m} v_{x x}+b v_{x x x t}=0 .
$$

The authors claimed that the conserved vectors constructed by partial Noether's theorem failed to satisfy the divergence relation and need to be adjusted to satisfy the divergence relationship. For the "partial Noether approach," in [13] Sarlet had given some negative comments (see Section 4 in [13]). So we will give some conservation laws of (1) by other methods.

In this paper, we investigate the Lagrangian and the selfadjointness and construct some new conservation laws for the generalized regularized long-wave equation by Ibragimov's theorem [14]. We should point out that the exact Lagrangian of (1) obtained in Section 2 is a nonlocal Lagrangian with an auxiliary function $\Psi$. In Section 3, we show that (1) is strictly self-adjoint, (2) is nonlinearly self-adjoint, and the minimal order of the differential substitution of (2) is equal to one. 
Based on these facts, some new conservation laws for the GRLW equation (1) are derived.

\section{The Auxiliary Lagrangian}

In this section, we will study the Lagrangian for the generalized regularized long-wave equation (1). First, we briefly present some notations to be used in what follows. Let $x=$ $\left(x_{1}, x_{2}, \ldots, x_{n}\right)$ be $n$ independent variables, let $u=u(x)$ be dependent variables, let

$$
D_{i}=\frac{\partial}{\partial x_{i}}+u_{i} \frac{\partial}{\partial u}+u_{i j} \frac{\partial}{\partial u_{i}}+\cdots
$$

be the total differentiation operator, and let

$$
\frac{\delta}{\delta u}=\frac{\partial}{\partial u}+\sum_{m \geq 1}(-1)^{m} D_{i_{1}} \cdots D_{i_{m}} \frac{\partial}{\partial u_{i_{1} \cdots i_{m}}}
$$

denote the Euler-Lagrange operator.

It is well known that a partial differential equation with odd order does not often admit a Lagrangian. However, noting the structure of (1), we can rewrite the equation as

$$
\left[u+b u_{x x}\right]_{t}+\left[u+\frac{a}{m+1} u^{m+1}\right]_{x}=0
$$

thus we find an auxiliary Lagrangian of (1) by the semiinverse method [15]. Consequently some conserved quantities of (1) can be derived from Noether's theorem.

According to (5), we introduce an auxiliary function $\Psi$ defined as

$$
\begin{gathered}
\Psi_{x}=u+b u_{x x}, \\
\Psi_{t}=-\left(u+\frac{a}{m+1} u^{m+1}\right),
\end{gathered}
$$

so that (5) is automatically satisfied.

By the semi-inverse method [15], we construct a trial Lagrangian in the form

$$
L(u, \Psi)=\left(u+b u_{x x}\right) \Psi_{t}-\left(u+\frac{a}{m+1} u^{m+1}\right) \Psi_{x}+F,
$$

where $F$ is an unknown function to be determined. The EulerLagrange equations of (8) are

$$
\begin{gathered}
-D_{t}\left(u+b u_{x x}\right)+D_{x}\left(u+\frac{a}{m+1} u^{m+1}\right)+\frac{\delta F}{\delta \Psi}=0, \\
\Psi_{t}+b D_{x}^{2} \Psi_{t}-\Psi_{x}\left(1+a u^{m}\right)+\frac{\delta F}{\delta u}=0 .
\end{gathered}
$$

Substituting (6) and (7) into the above equations, we obtain that

$$
\begin{gathered}
\frac{\delta F}{\delta \Psi}=2 \Psi_{x t}, \\
\frac{\delta F}{\delta u}=2 u+\frac{a(m+2)}{m+1} u^{m+1}+2 b\left(1+a u^{m}\right) u_{x x} \\
+a b m u^{m-1} u_{x}^{2} .
\end{gathered}
$$

Thus, we can take $F$ as

$$
F=-\Psi_{x} \Psi_{t}+u^{2}+\frac{a}{m+1} u^{m+2}-b\left(1+a u^{m}\right) u_{x}^{2}
$$

which implies that the Lagrangian of (1) reads

$$
\begin{aligned}
L= & \left(u+b u_{x x}\right) \Psi_{t}-\left(u+\frac{a u^{m+1}}{m+1}\right) \Psi_{x}-\Psi_{x} \Psi_{t} \\
& +u^{2}\left(1+\frac{a u^{m}}{m+1}\right)-b\left(1+a u^{m}\right) u_{x}^{2},
\end{aligned}
$$

which is subjected to (6). Therefore, we demonstrate the following result.

Theorem 1. Equation (1) admits a Lagrangian as follows:

$$
\begin{aligned}
L= & \left(u+b u_{x x}\right) \Psi_{t}-\left(u+\frac{a u^{m+1}}{m+1}\right) \Psi_{x}-\Psi_{x} \Psi_{t} \\
& +u^{2}\left(1+\frac{a u^{m}}{m+1}\right)-b\left(1+a u^{m}\right) u_{x}^{2}
\end{aligned}
$$

with constraint $\Psi_{x}=u+b u_{x x}$.

Proof. A direct calculation yields the Euler-Lagrangian equations of $L(u, \Psi)$ as follows:

$$
\begin{gathered}
-D_{t}\left(u+b u_{x x}\right)+D_{x}\left(u+\frac{a}{m+1} u^{m+1}\right)+2 \Psi_{x t}=0 \\
\Psi_{t}-\left(1+a u^{m}\right) \Psi_{x}+2 u+\frac{a(m+2)}{m+1} u^{m+1} \\
+m a b u^{m-1} u_{x}^{2}+b \Psi_{t x x} \\
+2 b\left(1+a u^{m}\right) u_{x x}=0 .
\end{gathered}
$$

Considering the constraint equation (6), it is obvious that (14) is equivalent to (5) and (15) can be rewritten in the form of

$$
\left(\Psi_{t}+u+\frac{a}{m+1} u^{m+1}\right)+b\left(\Psi_{t}+u+\frac{a}{m+1} u^{m+1}\right)_{x x}=0 .
$$

Solving the last equation we obtain that

$$
\Psi_{t}=-u-\frac{a}{m+1} u^{m+1}+P
$$

where $P=P(x, t)$ satisfies

$$
b P_{x x}+P=0 .
$$

Noting that $\Psi_{x}=u+b u_{x x}$, we deduce from $\Psi_{x t}=\Psi_{t x}$ that

$$
u_{t}+b u_{x x t}+u_{x}+a u^{m} u_{x}-P_{x}=0
$$

which combining with (5) yields $P_{x}=0$. Thus (18) implies $P=0$ and (17) implies that (7) holds.

Remark 2. The Lagrangian (13) of (1) with constraint (6) implies that auxiliary equation (7) holds. 


\section{Self-Adjointness and Conservation Laws}

In this section, we will investigate the self-adjointness and conservation laws of (1) by Ibragimov's theorem.

3.1. Self-Adjointness for (1) and (2). Let

$$
H=u_{t}+u_{x}+a u^{m} u_{x}+b u_{x x t} ;
$$

then we have the following formal Lagrangian for (1):

$$
\mathscr{L}_{1}=w\left(u_{t}+u_{x}+a u^{m} u_{x}+b u_{x x t}\right),
$$

where $w=w(x, t)$ is a new function. Computing the variational derivative of this formal Lagrangian, we obtain the following adjoint equation of (1):

$$
H^{*}=-w_{t}-w_{x}-a w_{x} u^{m}-b w_{x x t}=0 .
$$

Assume that $\left.H^{*}\right|_{w=\phi(t, x, u)}=\lambda H$, for a certain function $\lambda$. Then it is easy to obtain that $\phi=c_{1} u+c_{2}$ and $\lambda=-\phi_{u}=-c_{1}$, where $c_{1}, c_{2}$ are two arbitrary constants. This implies that (1) is quasiself-adjoint; especially, if $c_{1}=1$ and $c_{2}=0$; that is, $\phi=u$, then (1) is strictly self-adjoint (see [16] for the definitions). Thus, we have demonstrated the following statement.

Theorem 3. Equation (1) is quasiself-adjoint with the substitution $w=c_{1} u+c_{2}$, where $c_{1} \neq 0, c_{2}$ are two arbitrary constants. Especially, (1) is strictly self-adjoint with the substitution $w=u$.

Next, we discuss the self-adjointness of (2), and then by the nonlinear self-adjointness and Ibragimov's theorem on conservation laws we construct some conserved quantities of (1).

Similar to the above, we set

$$
E=v_{x t}+v_{x x}+a v_{x}^{m} v_{x x}+b v_{x x x t}
$$

then the formal Lagrangian for (2) is

$$
\mathscr{L}_{2}=w\left(v_{x t}+v_{x x}+a v_{x}^{m} v_{x x}+b v_{x x x t}\right)
$$

and the adjoint equation of (1) is

$$
E^{*}=w_{x t}+w_{x x}+a w_{x x} v_{x}^{m}+a m w_{x} v_{x}^{m-1} v_{x x}+b w_{x x x t}=0
$$

According to [16], (2) will be nonlinearly self-adjoint if there exists a differential substitution

$$
w=h\left(x, t, v, v_{x}, v_{t}, v_{x x}, \ldots,\right), \quad h \neq 0,
$$

involving a finite number of partial derivatives of $v$ with respect to $x$ and $t$, such that the equation

$$
\left.E^{*}\right|_{w=h}=\mu_{0} E+\mu_{1} D_{x} E+\mu_{2} D_{t} E+\mu_{3} D_{x}^{2} E+\cdots
$$

holds identically in the variables $t, x, u, u_{x}, u_{t}, \ldots$, where $\mu_{0}, \mu_{1}, \ldots$ are undetermined variable coefficients different from $\infty$ on the solutions of (2). The highest order of derivatives involved in $u$ is called the order of the differential substitution (26). The calculation provides the following result.
Theorem 4. Equation (2) is nonlinearly self-adjoint. The minimal order of the differential substitution (26) satisfying (27) is equal to one and is given by the function $h=c_{1} v_{x}+$ $c_{2} v_{t}+g(t)$, where $c_{1}$ and $c_{2}$ are two constants and $g(t)$ is a differentiable function of $t$.

3.2. Conservation Laws of (1). Now, we study the conservation laws of (1). We will first construct the conservation laws of (2) and then reduce them to the ones of (1). From the classical Lie group theory [17], we assume that a Lie point symmetry of (2) is a vector field

$$
X=\xi^{x}(t, x, v) \frac{\partial}{\partial x}+\xi^{t}(t, x, v) \frac{\partial}{\partial t}+\rho(t, x, v) \frac{\partial}{\partial v}
$$

on $\mathbb{R}^{+} \times \mathbb{R} \times \mathbb{R}$ such that $X^{(4)} E=0$ when $E=0$, where $E$ is given by (23). Taking into account (2), the operator $X^{(4)}$ is given as follows:

$$
X^{(4)}=X+\rho_{x}^{(1)} \frac{\partial}{\partial v_{x}}+\rho_{x t}^{(2)} \frac{\partial}{\partial v_{x t}}+\rho_{x x}^{(2)} \frac{\partial}{\partial v_{x x}}+\rho_{x x x t}^{(4)} \frac{\partial}{\partial v_{x x x t}},
$$

where

$$
\begin{gathered}
\rho_{x}^{(1)}=D_{x} \rho-\left(D_{x} \xi^{x}\right) v_{x}-\left(D_{x} \xi^{t}\right) v_{t}, \\
\rho_{x t}^{(2)}=D_{t} \rho_{x}^{(1)}-\left(D_{t} \xi^{x}\right) v_{x x}-\left(D_{t} \xi^{t}\right) v_{x t}, \\
\rho_{x x}^{(2)}=D_{x}\left(\rho_{x}^{(1)}\right)-\left(D_{x} \xi^{x}\right) v_{x x}-\left(D_{x} \xi^{t}\right) v_{x t}, \\
\rho_{x x x t}^{(4)}=D_{x}\left(\rho_{x x x}^{(3)}\right)-\left(D_{x} \xi^{x}\right) v_{x x x x}-\left(D_{x} \xi^{t}\right) v_{x x x t} .
\end{gathered}
$$

The condition $\left.X^{(4)} E\right|_{E=0}=0$ yields determining equations. Solving these determining equations, we can obtain the symmetries of (5) as follows:

$$
X_{1}=\frac{\partial}{\partial x}, \quad X_{2}=\frac{\partial}{\partial t}, \quad X_{3}=f(t) \frac{\partial}{\partial v}
$$

with $f(t)$ as an arbitrary function. Ibragimov's theorem on conservation laws yields the following conserved quantities of (2) for a general Lie symmetry $X=\xi^{x}(\partial / \partial x)+\xi^{t}(\partial / \partial t)+$ $\rho(\partial / \partial v)$. Consider the following:

$$
\begin{aligned}
C^{t}= & \xi^{t} \mathscr{L}_{2}-W\left(\frac{w_{x}}{2}+\frac{b w_{x x x}}{4}\right)+W_{x}\left(\frac{w}{2}+\frac{b w_{x x}}{4}\right) \\
& -W_{x x}\left(\frac{b w_{x}}{4}\right)+W_{x x x}\left(\frac{b w}{4}\right), \\
C^{x}= & \xi^{x} \mathscr{L}_{2}+W\left(a m v_{x}^{m-1} v_{x x} w-D_{x}\left[w\left(1+a v_{x}^{m}\right)\right]\right. \\
& \left.+\frac{w_{t}}{2}-\frac{3 b w_{x x t}}{4}\right) \\
& -W_{x}\left(\left(1+a v_{x}^{m}\right) w+\frac{b w_{x t}}{2}\right)+W_{t}\left(\frac{w}{2}+\frac{b w_{x x}}{4}\right) \\
& -W_{x t} \frac{b w_{x}}{2}+W_{x x t}\left(\frac{3 b w}{4}\right),
\end{aligned}
$$


where $W=\rho-\xi^{x} v_{x}-\xi^{t} v_{t}$ is the Lie characteristic function and $w$ is given by the differential substitution $w=c_{1} v_{x}+c_{2} v_{t}+$ $g(t)$. Let us construct the conserved vector corresponding to the space translation group with the generator

$$
X_{1}=\frac{\partial}{\partial x}
$$

In this case $W=-v_{x}$. Taking account of the differential substitution $w=c_{1} v_{x}+c_{2} v_{t}+g(t)$ and $v_{x}=u$, from (32), we obtain the conserved quantities of (2), which can be transformed to the ones of (1) as follows:

$$
\begin{aligned}
C_{1}^{t}= & \frac{1}{4} c_{2}\left(2 u u_{t}+b u u_{x x t}-2 u_{x} \int u_{t} d x\right. \\
& \left.\quad-b u_{x} u_{x t}+b u_{t} u_{x x}-b u_{x x x} \int u_{t} d x\right) \\
& -\frac{1}{4} b g(t) u_{x x x}-\frac{1}{2} g(t) u_{x} \\
= & \frac{1}{2} c_{2}\left(2 u u_{t}+b u u_{x x t}+b u_{t} u_{x x}\right) \\
& -\frac{1}{4} c_{2} D_{x}\left(b u u_{x t}+2 u \int u_{t} d x+b u_{x x} \int u_{t} d x\right) \\
& -\frac{1}{4} D_{x}\left[b g(t) u_{x x}+2 g(t) u\right] \\
& +\frac{1}{4} c_{2}\left(3 b u u_{x t t}-2 b u_{x} u_{t t}+b u_{t} u_{x t}+b u_{x x} \int u_{t t} d x\right. \\
& +\frac{1}{4} D_{t}\left[b g(t) u_{x x}+2 g(t) u\right] \\
& +\frac{1}{4} c_{2} D_{t}\left(b u u_{x t}+2 u \int u_{t} d x+b u_{x x} \int u_{t} d x\right) \\
& +c_{1} u\left(u_{x}+u_{t}+a u^{m} u_{x}+b u_{x x t}\right)+\frac{1}{2}\left[u g^{\prime}(t)+g(t) u_{t}\right] \\
& +\frac{b}{4}\left[g(t) u_{x x t}+u_{x}(t) u_{x x}+4 a u^{m+1} u_{t}\right. \\
& \left.+4 u u_{t}+2 u_{t} \int u_{t} d x+b u_{x x t} \int u_{t} d x\right)
\end{aligned}
$$

which can be reduced to the form

$$
\begin{gathered}
C_{1}^{t}=\frac{1}{2} c_{2}\left(2 u u_{t}+b u u_{x x t}+b u_{t} u_{x x}\right), \\
C_{1}^{x}=\frac{c_{2}}{2}\left(b u u_{x t t}-b u_{x} u_{t t}+2 a u^{m+1} u_{t}+2 u u_{t}\right) .
\end{gathered}
$$

Here we have used (1).
By the procedure analogous to that used above, we obtain the conserved quantities of (2) corresponding to the generators $X_{2}$ and $X_{3}$, which can be reduced by the differential substitutions $w=c_{1} v_{x}+c_{2} v_{t}+g(t)$ and $v_{x}=u$ to the conserved quantities of (1) as follows:

$$
\begin{aligned}
C_{2}^{t}= & \frac{c_{1} b}{2}\left(u u_{x x t}-u_{t} u_{x x}\right)+a g(t) u^{m} u_{x}, \\
C_{2}^{x}= & \frac{c_{1} b}{2}\left(u_{x} u_{t t}-u u_{x t t}\right)-a g(t) u^{m} u_{t} \\
& +g^{\prime}(t)\left(\int u_{t} d x+u+b u_{x t}\right), \\
C_{3}^{t}= & f(t)\left(c_{1} u_{x}+c_{2} u_{t}\right), \\
C_{3}^{x}= & f(t)\left(c_{1} u_{x}+c_{2} u_{t}\right)\left(1+a u^{m}\right) \\
& +b f(t)\left(c_{1} u_{x x t}+c_{2} u_{x t t}\right) \\
& -f^{\prime}(t)\left(c_{1} u+c_{2} \int u_{t} d x+g(t)\right) .
\end{aligned}
$$

Here we have used (1).

Remark 5. In fact, (2) admits a Lagrangian

$$
L_{2}=-\frac{1}{2} v_{x} v_{t}-\frac{1}{2} v_{x}^{2}-\frac{a}{(m+1)(m+2)} v_{x}^{m+2}+\frac{b}{2} v_{x t} v_{x x}
$$

and has the following Noether symmetry generators:

$$
\begin{gathered}
X_{5}=\frac{\partial}{\partial x}, \quad X_{6}=\frac{\partial}{\partial t}, \\
X_{7}=f(t) \frac{\partial}{\partial v} \quad \text { with gangue function } A^{t}=0, \\
A^{x}=-\frac{1}{2} f^{\prime}(t) v,
\end{gathered}
$$

where $f(t)$ is a differentiable function in $t$. Noether's theorem on conservation laws [18] can yield the conserved quantities of (2), which are the same as the ones in [11]. Those were constructed by the so-called "partial Noether approach."

\section{Conclusions}

In this paper, we investigate the Lagrangians and selfadjointnesses of the GRLW equation (1) and its transformed equation (2). We show that (1) has a nonlocal Lagrangian with an auxiliary function and is strictly self-adjoint; its transformed equation is nonlinearly self-adjoint and the minimal order of the differential substitution is equal to one. Then by Ibragimov's theorem on conservation laws we obtain some conserved qualities of the generalized regularized longwave equation.

\section{Conflict of Interests}

The authors declare that there is no conflict of interests regarding the publication of this paper. 


\section{Acknowledgments}

The first author is partly supported by Zhejiang Provincial Natural Science Foundation of China under Grant no. LY12A01003 and Subjects Research and Development Foundation of Hangzhou Dianzi University under Grant no. ZX100204004-6. The second author is partly supported by NSFC under Grant no. 11101111, and Zhejiang Provincial Natural Science Foundation of China under Grant no. LY14A010029.

\section{References}

[1] D. H. Peregrine, "Calculations of the development of an undular bore," Journal of Fluid Mechanics, vol. 25, no. 2, pp. 321-330, 1966.

[2] D. H. Peregrine, "Long waves on a beach," Journal of Fluid Mechanics, vol. 27, no. 4, pp. 815-827, 1967.

[3] M. Dehghan and R. Salehi, "The solitary wave solution of the two-dimensional regularized long-wave equation in fluids and plasmas," Computer Physics Communications, vol. 182, no. 12, pp. 2540-2549, 2011.

[4] J. C. Eilbeck and G. R. McGuire, "Numerical study of the regularized long-wave equation. II: interaction of solitary waves," Journal of Computational Physics, vol. 23, no. 1, pp. 63-73, 1977.

[5] M. E. Alexander and J. L. Morris, "Galerkin methods applied to some model equations for non-linear dispersive waves," Journal of Computational Physics, vol. 30, no. 3, pp. 428-451, 1979.

[6] Q. Chang, "Conservative scheme for a model of nonlinear dispersive waves and its solitary waves induced by boundary motion," Journal of Computational Physics, vol. 93, no. 2, pp. 360-375, 1991.

[7] D. Bhardwaj and R. Shankar, "Computational method for regularized long wave equation," Computers and Mathematics with Applications, vol. 40, no. 12, pp. 1397-1404, 2000.

[8] D. Kaya, "A numerical simulation of solitary-wave solutions of the generalized regularized long-wave equation," Applied Mathematics and Computation, vol. 149, no. 3, pp. 833-841, 2004.

[9] A.-M. Wazwaz, "A sine-cosine method for handling nonlinear wave equations," Mathematical and Computer Modelling, vol. 40, no. 5-6, pp. 499-508, 2004.

[10] A. Mohebbi, "Solitary wave solutions of the nonlinear generalized Pochhammer-Chree and regularized long wave equations," Nonlinear Dynamics, vol. 70, no. 4, pp. 2463-2474, 2012.

[11] R. Naz, M. D. Khan, and I. Naeem, "Conservation laws and exact solutions of a class of non linear regularized long wave equations via double reduction theory and Lie symmetries," Communications in Nonlinear Science and Numerical Simulation, vol. 18, no. 4, pp. 826-834, 2013.

[12] A. Biswas, "Solitary waves for power-law regularized long-wave equation and $R(m, n)$ equation," Nonlinear Dynamics, vol. 59, no. 3, pp. 423-426, 2010.

[13] W. Sarlet, "Comment on 'conservation laws of higher order nonlinear PDEs and the variational conservation laws in the class with mixed derivatives,' Journal of Physics A: Mathematical and Theoretical, vol. 43, no. 45, Article ID 458001, 2010.

[14] N. H. Ibragimov, "A new conservation theorem," Journal of Mathematical Analysis and Applications, vol. 333, no. 1, pp. 311328, 2007.
[15] J.-H. He, "Variational principles for some nonlinear partial differential equations with variable coefficients," Chaos, Solitons and Fractals, vol. 19, no. 4, pp. 847-851, 2004.

[16] N. H. Ibragimov, "Nonlinear self-adjointness and conservation laws," Journal of Physics A: Mathematical and Theoretical, vol. 44, no. 43, Article ID 432002, 2011.

[17] P. J. Olver, Application of Lie Groups To Differential Equations, Springer, Berlin, Germany, 1986.

[18] E. Noether, "Invariante variationsprobleme," Nachrichten von der Gesellschaft der Wissenschaften zu Göttingen, MathematischPhysikalische Klasse, vol. 2, pp. 235-257, 1918. 


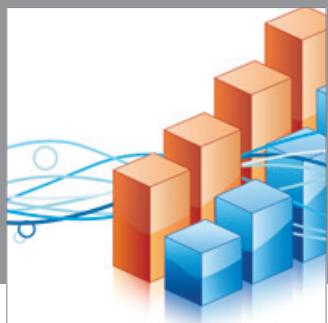

Advances in

Operations Research

mansans

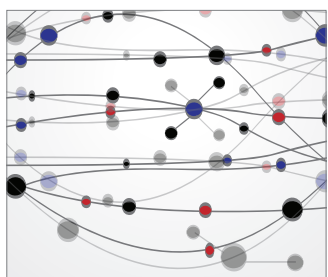

The Scientific World Journal
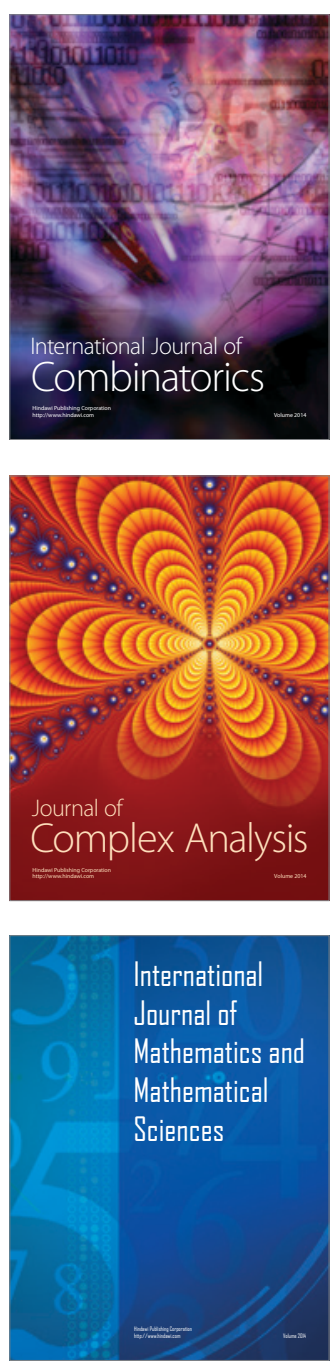
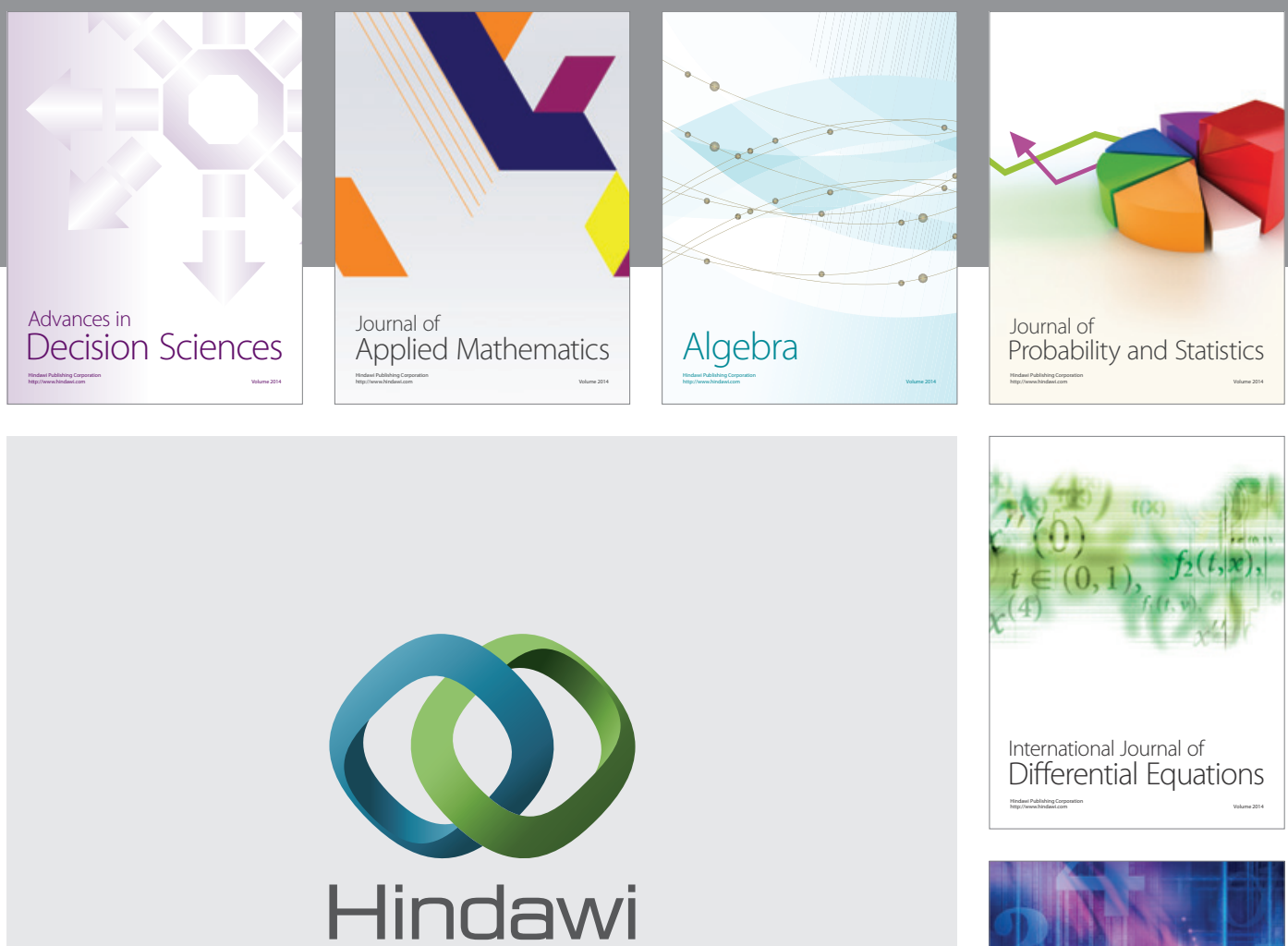

Submit your manuscripts at http://www.hindawi.com
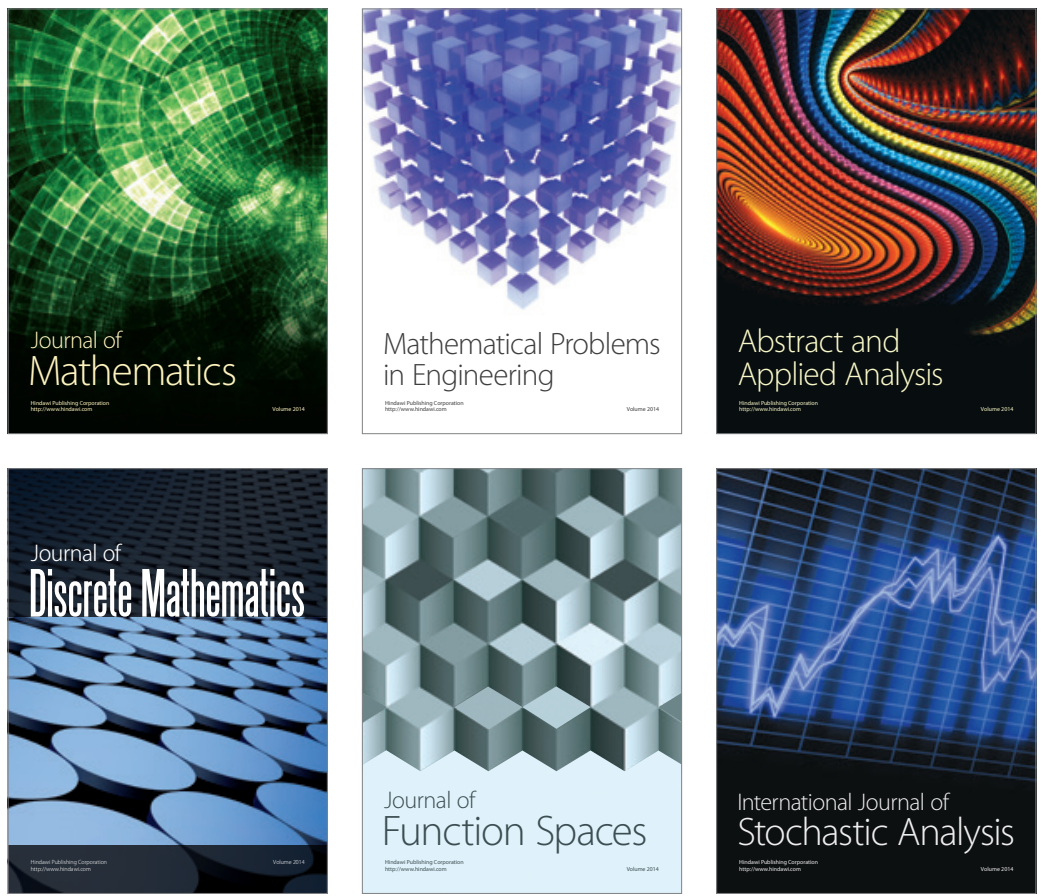

Journal of

Function Spaces

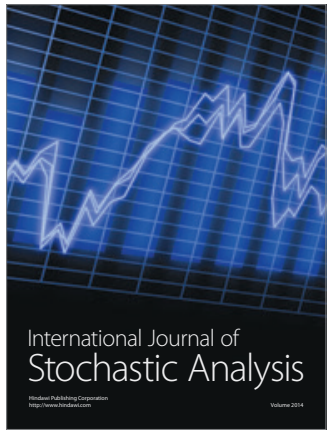

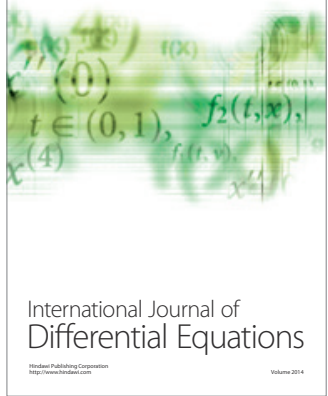
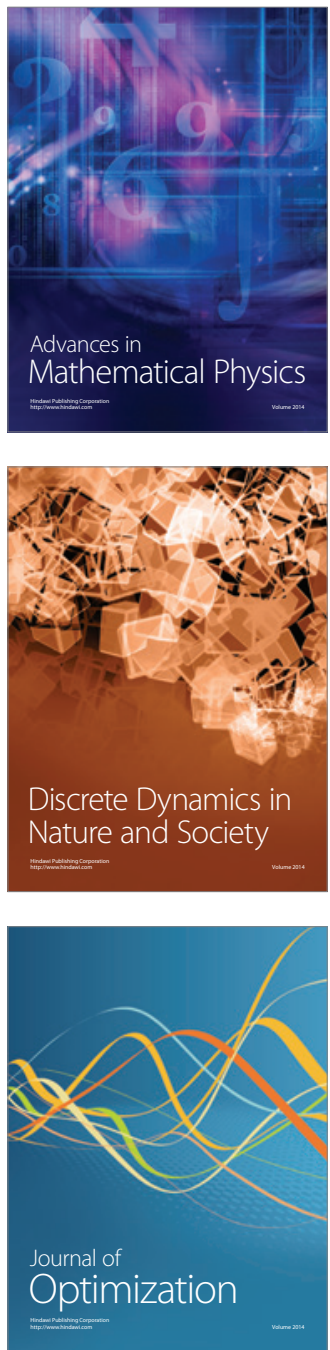